\title{
Study on Direct Writing of Gallium Metal for the Flexible Sensor
}

\author{
Junfeng Liu, ${ }^{1}$ Haopeng Ma, ${ }^{1}$ Yong Yang, ${ }^{2}$ Weimin Yang, ${ }^{1}$ Zhiwei Jiao $\mathbb{D}^{1},{ }^{1}$ and Yuan Yu $\mathbb{D}^{1}$ \\ ${ }^{1}$ College of Mechanical and Electrical Engineering, Beijing University of Chemical Technology, Beijing 100029, China \\ ${ }^{2}$ Ningbo Institute of Materials Technology and Engineering, CAS, Ningbo 315000, China
}

Correspondence should be addressed to Zhiwei Jiao; jiaozw@mail.buct.edu.cn and Yuan Yu; yuyuanjd@263.net

Received 15 March 2021; Accepted 17 September 2021; Published 6 October 2021

Academic Editor: Xuchun Gui

Copyright (c) 2021 Junfeng Liu et al. This is an open access article distributed under the Creative Commons Attribution License, which permits unrestricted use, distribution, and reproduction in any medium, provided the original work is properly cited.

There is an urgent need for a simple and effective method to manufacture flexible sensors composed of liquid metal. Gallium (Ga) metal has become an ideal flexible conductive material due to its high conductivity, low melting point, and high flow characteristics. In this paper, liquid Ga metal is directly written on the polyvinyl alcohol (PVA) film through the driving mode of piston extrusion; then, the Ga metal wire is transferred and sealed with silica gel. The advantages of piston mode are studied, and the direct writing parameters of the liquid Ga metal, including extrusion speed, nozzle height, printing speed, and nozzle inner diameter, are systematically optimized. The flexible sensor based on the sealed liquid Ga metal has good resilience under the external load. This work provides a specific reference for direct writing of liquid Ga metal and its sealing technology for the flexible sensor.

\section{Introduction}

Nowadays, the application environment of electronic products is becoming more and more complex. Flexible sensors, due to the flexibility, short response time, high sensitivity, and features of sensing the force on the surface of objects, have drawn extensive attention [1]. Flexible sensors have a wide range of applications in wearable sensors [2-5], health monitoring devices [6-11], and soft robotics [12, 13]. For instance, Marques et al. designed and developed a muscular monitoring patch, which has further development of multilayer biomonitoring applications [5]. Yu et al. presented a flexible pressure sensor constructed by threedimensional polypyrene foam, which can detect carcinoembryonic antigen sensitively and rapidly [6]. For the flexible sensors, not only the functional requirements of products are important but also the flexibility and ductility requirements of electronic products are highly emphasized. The rapid development on flexible substrates and liquid metals has largely enriched the field of the flexible sensors $[14,15]$. Gallium-based liquid metals, with good mechanical properties and excellent conductivity, are a reliable choice for making flexible electronic equipment and flexible sensors [16-19].
As a metal with low melting point (melting point: $29.8^{\circ} \mathrm{C}$ ), liquid $\mathrm{Ga}$ metal has the advantages of high conductivity $\left(29 \times 10^{-6} \Omega \mathrm{cm}\right)$ [20], low chemical activity, high fluidity (2.4 $\mathrm{mPa} \mathrm{s})$ [21], and low toxicity. However, liquid Ga metal has a very high surface tension $\left(0.5-0.72 \mathrm{~N} \mathrm{~m}^{-1}\right)$. The external pressure applied on the liquid Ga metal must reach a certain value to make the liquid $\mathrm{Ga}$ metal exhibit fluidity, which will hinder the forming process [22]. In recent years, liquid metal forming methods are mainly focused on mask deposition, microchannel injection, and direct writing. Fassler et al. used mask deposition method to pour liquid metal into a mold and then scraped off excess metal [23].

The prepared liquid metal pattern was sealed in a vacuum environment to fabricate flexible devices. Jin et al. injected the sealing material (such as gelatin) biocompatible with liquid metal into the biological tissue to form a specific structure [24]. Then, a tool (such as an injection needle) was inserted, and the target region was pulled out to shape a mold of electrode. Finally, the conductive metal ink, the insulating ink, and matching micro nano scale devices were successively injected to form the target electronic device. Liu et al. developed a 3D desktop automatic printing equipment, which can even directly write paper-based functional 
electronic circuits in normal temperature [25]. In the mask deposition method and the microchannel injection method, high-precision lithography equipment and a vacuum processing environment are required. The technology cost is relatively high, and there are still some problems such as low accuracy and poor stability. However, the direct writing method requires oxidation of the liquid metal to reduce the fluidity and surface tension. After the oxidation of liquid $\mathrm{Ga}$ metal, it will bring some obstacles to forming precision and quality, and the resistivity of printed stuff will increase [26].

After the direct writing, the liquid metal needs to be stripped from substrate and sealed in order to meet the application requirements. However, the liquid metal wire cannot be stripped perfectly from the rigid substrate without damage. Moreover, Ga metal structure will be damaged easily in the sealing process. For example, Lee et al. poured the polydimethylsiloxane (PDMS) solution directly on the metal circuit for curing and sealing $[27,28]$. However, the cured PDMS is brittle and it is difficult to peel the Ga metal structure from the substrate, which will affect the precision and even destroy the liquid metal circuit. Research studies show that the liquid metal has a good biocompatibility with PVA film. The PVA film is often used in the field of transfer printing $[18,29-31]$. What is more attractive is that the PVA material has a strong water-soluble property. The damage caused by removing metal structure from PVA substrate is much less than that of other films. Therefore, PVA film is selected as the flexible substrate for direct writing of liquid Ga metal.

In view of above problems, the direct writing technology of liquid metal is studied in this paper. The precise forming technology of unoxidized liquid $\mathrm{Ga}$ metal is realized based on systematic study of the forming process. Based on the water-soluble properties of PVA film, a transferring and sealing technology for direct writing of liquid Ga metal on the PVA film is proposed. Experimental results show that the prepared flexible electronic sensor based on the sealed liquid Ga metal possesses good resilience and sensitive response performance, which can be used under extreme deformation conditions due to its self-recovering ability.

\section{Materials and Methods}

2.1. Materials. Ga (purity of 99.999) was obtained from Runde Metal Materials Co., Ltd., and its melting point is $29.8^{\circ} \mathrm{C}$, density is $5.904 \mathrm{~g} / \mathrm{cm} 3$, and surface tension is $700 \mathrm{mN} / \mathrm{m}$.

The average particle size of PVA powder is $150 \mu \mathrm{m}$ (produced by Chinese Shanghai Chenqi Environmental Protection Raw Material Company).

Ecoflex-0030 silica gel was obtained from Smooth-On, Inc., which consists of component $\mathrm{A}$ and component $\mathrm{B}$.

2.2. Preparation. The specific experimental technology is depicted in Figure 1. (1) PVA powder and pure water are mixed in a weight ratio of $1: 9$ and then stirred magnetically until the solution becomes transparent. (2) The PVA solution with a thickness of $0.3 \mathrm{~mm}$ is uniformly coated on the surface of PMMA substrate and dried. (3) Liquid Ga metal is directly written on PMMA substrate with PVA film on the surface. (4) Component A and component B of Ecoflex-0030 silica gel are mixed in a weight ratio of $1: 1$ and poured on the liquid $\mathrm{Ga}$ metal structure. (5) The liquid Ga metal covered by silica gel is removed from the PMMA substrate. (6) The sealed product is immersed in pure water for 5 minutes, and then the PVA film is torn off. (7) The other side of the film is sealed using the same method.

\section{Results and Discussion}

The width of Ga wire is measured by optical microscope. The resistance of $\mathrm{Ga}$ wire is measured with a multimeter. The printing parameters have an important effect on the forming quality of the liquid Ga metal, and the key parameters in the forming technology and their setting range in the experiments are listed in Table 1.

\subsection{Driving Mode of Direct Writing for the Ga Metal.} Currently, the most common methods for extruding fluid include screw extrusion, pneumatic extrusion, and piston extrusion. The single-screw extruder is taken as an example. The transportation of materials mainly depends on the friction between the materials, the screw, and the inner wall of the barrel. There are certain requirements for the friction coefficient and viscosity. The single-screw extruder has the defects of unstable extrusion flow and pressure. In addition, the corrosive effect of liquid Ga metal on other metal materials will make the selection of screws and melt pumps more difficult. Therefore, screw extrusion is not suitable for liquid metal.

In order to explore whether pneumatic extrusion and piston extrusion are suitable for direct writing of liquid $\mathrm{Ga}$ metal, a pneumatic extrusion and a piston extrusion platform are constructed. The results of pneumatic extrusion and piston extrusion are shown in Figure 2. The Ga metal wire of pneumatic extrusion is shown in Figure 2(a). The Ga wire is not smooth, and there are obvious "burrs." The Ga metal wire of piston extrusion is shown in Figure 2(b), and the $\mathrm{Ga}$ metal wire is smooth and even relatively.

It is considered that the liquid Ga metal driven by pneumatic extrusion can be squeezed and printed. However, there are three disadvantages. (1) The flow rate of extrusion is unstable. There is a collision near the interface of the two fluids, and the resulting turbulence further destroys the stability of flow rate. (2) The extrusion speed is difficult to control accurately. The extrusion of liquid Ga metal requires a certain amount of pressure. However, it is difficult to control the extrusion rate of liquid Ga metal with good fluidity under high-pressure gas. It affects the forming accuracy to a large extent. (3) The response of the system lags behind. The pressure value adjustment needs several steps, which causes delayed output response.

There are two advantages of the piston extrusion. (1) The extrusion flow rate is stable. The extrusion volume is only determined by the stroke of the piston and is not limited by other factors. (2) Motion control is accurate. The extrusion 

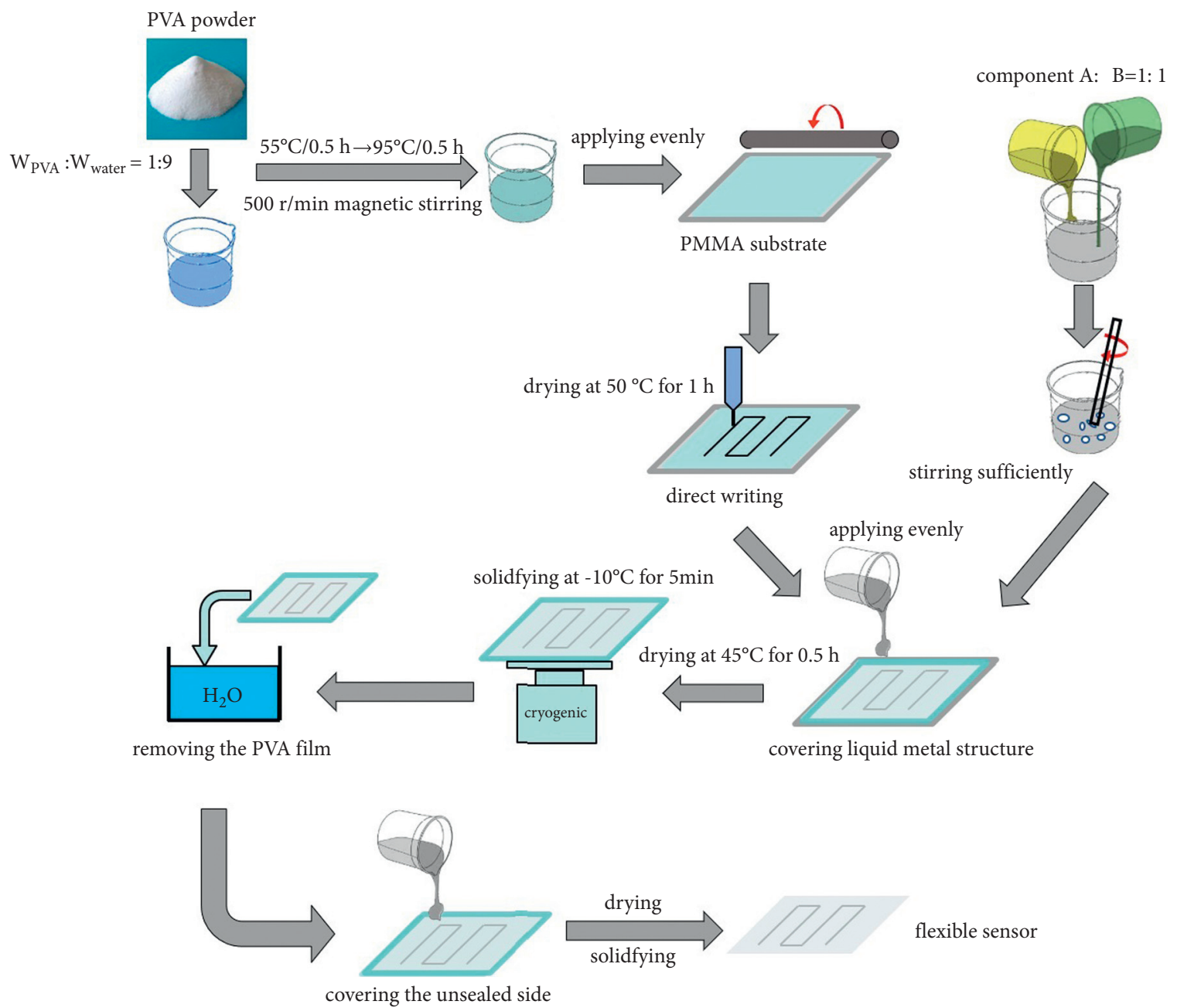

Figure 1: Manufacturing technology of the liquid Ga metal flexible sensor.

TABle 1: Parameters in the direct writing of liquid Ga metal.

\begin{tabular}{lccc}
\hline Parameter & Symbol & Unit & Value range \\
\hline Extrusion speed & $Q$ & $\mathrm{~mm}^{3} / \mathrm{s}$ & $1-3$ \\
Nozzle height & $h$ & $\mathrm{~mm}$ & $0.05-0.25$ \\
Printing speed & $v$ & $\mathrm{~mm} / \mathrm{s}$ & $15-65$ \\
Printing temperature & $T$ & ${ }^{\circ} \mathrm{C}$ & 40 \\
Width of Ga wire & $W$ & $\mathrm{~mm}$ & Dependent variable \\
Nozzle inner diameter & $d$ & $\mathrm{~mm}$ & $0.11-0.61$ \\
\hline
\end{tabular}

system is a closed loop so that response is timely. The extrusion volume can be controlled in real time according to the application demand.

Therefore, it is comprehensively determined that the pneumatic extrusion method is not suitable for the liquid metal printing and forming. In this study, the piston extrusion is selected as the driving mode.

3.2. Effects of Extrusion Speed on the Forming Quality of the Ga Metal. The extrusion speed $Q$ is set to $1 \mathrm{~mm}^{3} \cdot \mathrm{s}^{-1}$, $1.5 \mathrm{~mm}^{3} \cdot \mathrm{s}^{-1}, \quad 2 \mathrm{~mm}^{3} \cdot \mathrm{s}^{-1}, \quad 2.5 \mathrm{~mm}^{3} \cdot \mathrm{s}^{-1}$, and $3 \mathrm{~mm}^{3} \cdot \mathrm{s}^{-1}$, nozzle height $h=0.1 \mathrm{~mm}$, nozzle inner diameter $d=0.61 \mathrm{~mm}$, printing speed $v=40 \mathrm{~mm} \cdot \mathrm{s}^{-1}$, and printing temperature $T=40^{\circ} \mathrm{C}$. The effect of extrusion speed on the Ga metal wire width is shown in Figure 3(a). It is observed that $Q=2 \mathrm{~mm}^{3} / \mathrm{s}$ is the inflection point of $W$ value fitting curve. When $Q<2 \mathrm{~mm}^{3} / \mathrm{s}, W$ value increases with the increase of $Q$ value; when $Q>2 \mathrm{~mm}^{3} \cdot \mathrm{s}^{-1}$, the value of $W$ decreases. The standard deviation becomes large, which illustrates that the Ga wire size fluctuates drastically and the stability decreases. It is considered that the extrusion amount is larger than necessary one, which causes the liquid Ga metal to accumulate locally in the forming path. Affected by the surface tension of $\mathrm{Ga}$ metal, the liquid Ga metal near the accumulated point moves closer to the stacking point. 


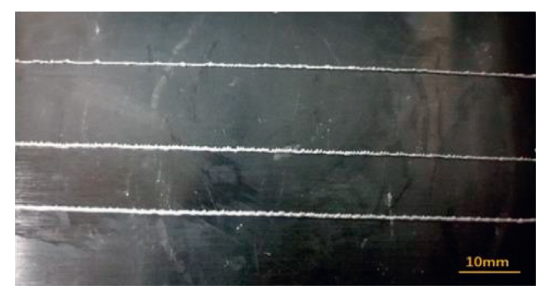

(a)

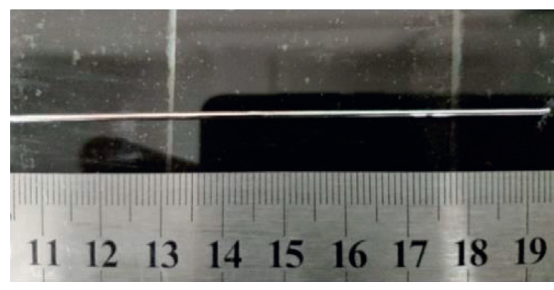

(b)

Figure 2: Direct writing of Ga metal wire with different extrusion methods. (a) Ga wire by direct writing of pneumatic extrusion. (b) Ga wire by direct writing of piston extrusion.

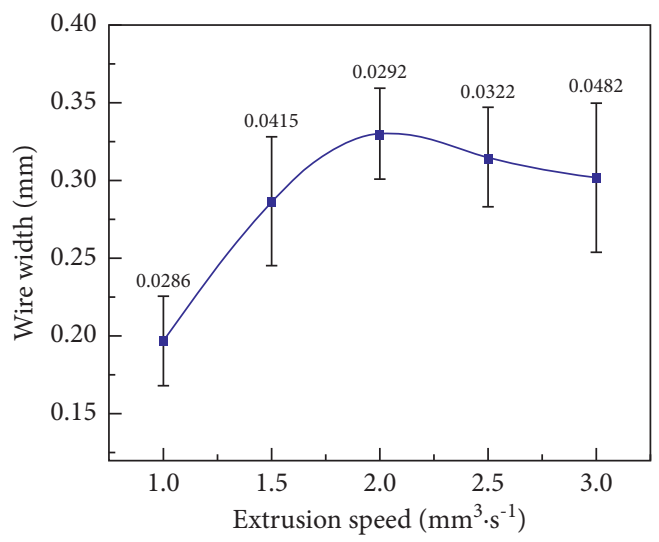

(a)

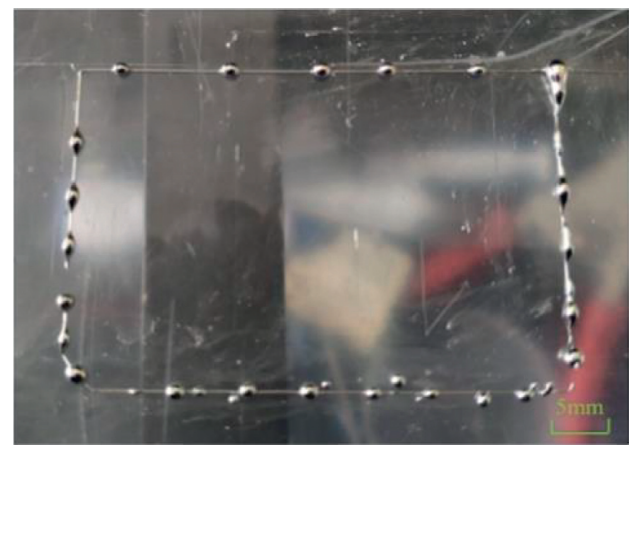

(b)

Figure 3: Effects of extrusion speed on forming quality. (a) Effect of extrusion speed on the wire width. (b) Ga metal stacking points.

Therefore, the volume of the stacking point is further enlarged, and the wire width $W$ around the stacking point becomes small. The faster the extrusion speed is, the more the stacking points are. When $Q=3 \mathrm{~mm}^{3} \cdot \mathrm{s}^{-1}$, the corresponding formed $W=0.302 \mathrm{~mm}$, and wire width standard deviation is $0.0482 \mathrm{~mm}$. With the further increase of the extrusion speed $Q$, the $G$ a wire will not meet the requirements of use. The printed Ga metal wires are depicted in Figure 3(b). Considering the stability of Ga metal forming, it is determined that the most suitable extrusion speed is $2 \mathrm{~mm}^{3} \cdot \mathrm{s}^{-1}$ when the nozzle inner diameter is $0.61 \mathrm{~mm}$.

\subsection{Effects of Nozzle Height on the Forming Quality of the $\mathrm{Ga}$} Metal. According to the conclusions in the previous sections, the extrusion speed $Q=2 \mathrm{~mm}^{3} \cdot \mathrm{s}^{-1}$. In the same way, printing speed of $v=40 \mathrm{~mm} \cdot \mathrm{s}^{-1}$, nozzle inner diameter of $d=0.61 \mathrm{~mm}$, and printing temperature of $T=40^{\circ} \mathrm{C}$ are set. Between $0.05 \mathrm{~mm}$ and $0.25 \mathrm{~mm}, 11$ points are selected as the height of nozzle based on the $0.02 \mathrm{~mm}$ spacing. The effects of nozzle height on the Ga metal wire width are shown in Figure 4. It is known that the difference of the nozzle height leads to different flows of liquid Ga metal between the nozzle and the substrate. Taking the Ga metal in the contact portion between the nozzle outlet and the substrate as the stress object, the main forces acting on this Ga metal unit affecting its formation include the following. (1) Vertical downward force generated by extrusion pressure. (2) Adhesion between substrate and Ga metal printed on substrate. (3) The surface tension of Ga metal. (4) When the nozzle drives the liquid Ga metal to move at a certain velocity, the oxide film on the surface will shear the Ga metal at the nozzle. The shear force will drag Ga metal, which is referred to as the internal force. (5) The gravity of the Ga metal unit. When $h \leq 0.05 \mathrm{~mm}$, the printed Ga metal wires are shown in Figures 5(a) and 5(b). At this time, the space between the nozzle and the substrate is narrow. The adhesion is not enough to overcome the surface tension of the Ga metal. The Ga metal unit at the nozzle moves with the liquid metal in the nozzle at a certain velocity, and it cannot be deposited into wire. Figure 5(c) shows the printed Ga metal wire when the nozzle height is $0.05 \mathrm{~mm}<h<0.13 \mathrm{~mm}$. With the increase of nozzle height, the necking phenomenon of Ga metal occurs. Ga metal breaks through the limitation of surface tension under the action of adhesion force and deposits on the substrate. At this time, the shear force overcomes the surface tension of $\mathrm{Ga}$ metal, and the Ga metal unit at the nozzle changes its morphology, which is deposited on the substrate. The flow rate gradually approaches the extrusion speed $Q$ needed for stable forming, but the forming is still unstable. When $h 0.13 \mathrm{~mm}$, the flow is basically equal to $Q$, and the wire forming is stable. The printing result of the Ga metal wire is shown in Figure 5(d). With the increase of $h$, Ga metal will be deposited on the forming platform in the form of droplet transition, which is mainly affected by gravity. Although the liquid metal extruded can be wired to the substrate, the 


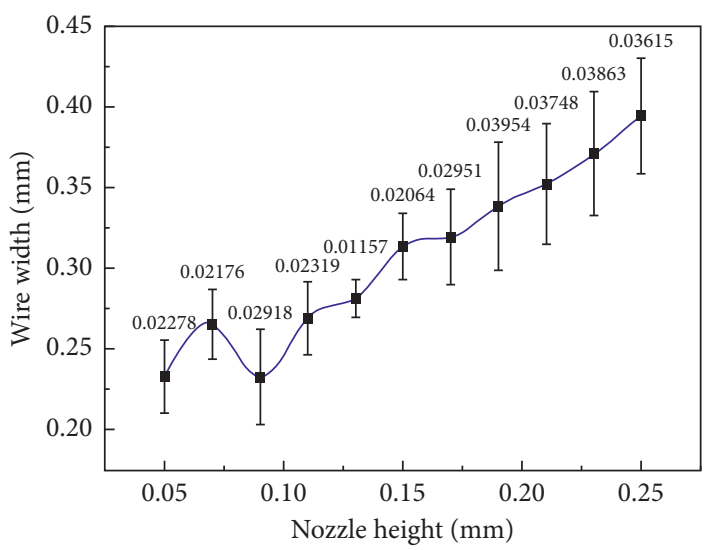

FIgURE 4: The effects of nozzle height on the Ga metal wire width.
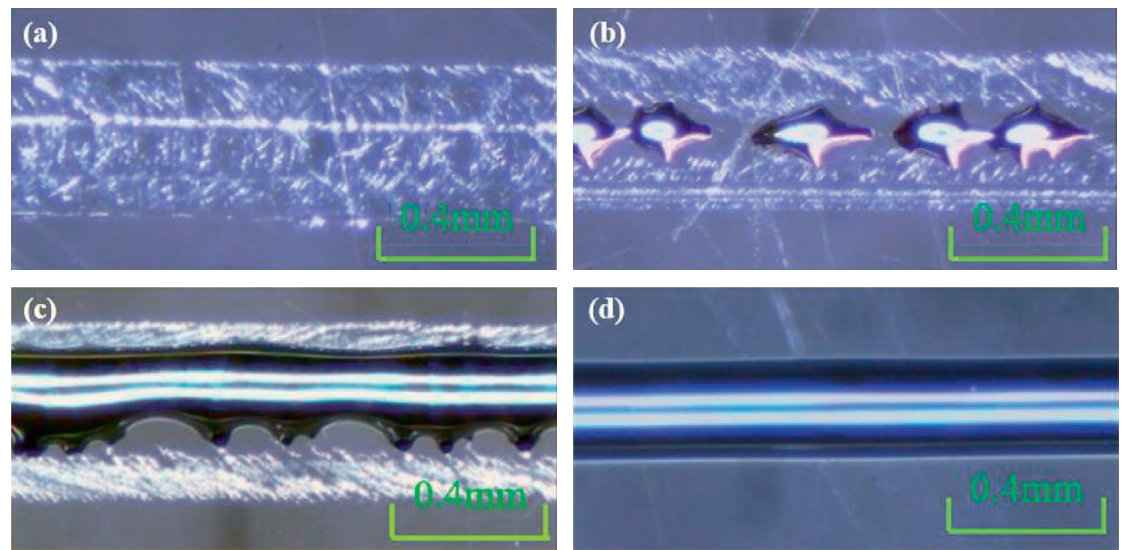

FIGURE 5: Direct writing of Ga metal under different nozzle heights. (a) $h=0.01 \mathrm{~mm}$. (b) $\mathrm{h}=0.03 \mathrm{~mm}$. (c) $0.05 \mathrm{~mm} \leq h<0.13 \mathrm{~mm}$. (d) $\mathrm{h} \approx$ $0.13 \mathrm{~mm}$.

standard deviation is relatively large and the stability is poor. Therefore, when the inner diameter of the nozzle is $0.61 \mathrm{~mm}$, the optimal height of the nozzle is $0.13 \mathrm{~mm}$.

\subsection{Effects of Printing Speed on the Forming Quality of the $\mathrm{Ga}$} Metal. According to the above two sections, the extrusion speed of $Q=2 \mathrm{~mm}^{3} \cdot \mathrm{s}^{-1}$, the nozzle height of $h=0.13 \mathrm{~mm}$, and the printing temperature of $T=40^{\circ} \mathrm{C}$ are determined. In the same way, the nozzle inner diameter $d$ is selected as $0.61 \mathrm{~mm}$. Between $15 \mathrm{~mm} \cdot \mathrm{s}^{-1}$ and $65 \mathrm{~mm} \cdot \mathrm{s}^{-1}, 11$ points are selected as the printing speed $v$ based on the $5 \mathrm{~mm} \cdot \mathrm{s}^{-1}$ spacing. The effects of printing speed on the Ga metal wire width are shown in Figure 6. The results show that the $W$ value is basically stable in the range of $0.32 \mathrm{~mm}-0.34 \mathrm{~mm}$ when $15 \mathrm{~mm} \cdot \mathrm{s}^{-1}<v<35 \mathrm{~mm} \cdot \mathrm{s}^{-1}$. However, the amount of extruded metal per unit length is larger than the required amount, so that the forming stability decreases and the fluctuation of $W$ and its standard deviation increase. When $v$ $\approx 40 \mathrm{~mm} \cdot \mathrm{s}^{-1}$, the wire formation is stable, the wire width is $0.278 \mathrm{~mm}$, and the standard deviation is $0.01267 \mathrm{~mm}$. When $40 \mathrm{~mm} \cdot \mathrm{s}^{-1}<v<55 \mathrm{~mm} \mathrm{~s} \mathrm{~s}^{-1}$, the value of $W$ decreases with the increase of $v$ and the standard deviation of $W$ remains relatively stable. When $v>55 \mathrm{~mm} / \mathrm{s}$, the extruded liquid metal will have no time to deposit on the surface of the printing substrate. The process of direct writing becomes unstable. $W$ continues to decrease with the increase of $v$ and breakpoints appear on the forming path, which make it difficult to prepare Ga metal wire stably. According to the above analysis, the optimal printing speed is $40 \mathrm{~mm} \cdot \mathrm{s}^{-1}$. The experimental results with the printing speed of $40 \mathrm{~mm} \cdot \mathrm{s}^{-1}$ are shown in Figure 7.

3.5. Effects of the Nozzle Inner Diameter on the Forming Quality of the Ga Metal. According to the experiments, if the nozzle inner diameter $d$ is small, for example, when it is $0.06 \mathrm{~mm}$, the extrusion resistance under the action of high surface tension of liquid $\mathrm{Ga}$ metal is too large so that the Ga metal wire is difficult to form. With the increase of nozzle diameter, liquid $\mathrm{Ga}$ metal can be extruded smoothly when $d=0.11 \mathrm{~mm}$. Therefore, it is determined that $d=0.11 \mathrm{~mm}$ is the minimum nozzle diameter available in the experiment. When $d=0.61 \mathrm{~mm}$, the maximum width of Ga metal wire can reach about $0.34 \mathrm{~mm}$ stably, which meets the application requirement of flexible sensors. Therefore, nozzles with different nozzle inner diameters $(d=0.11-0.61 \mathrm{~mm})$ are used for printing in the experiments. Thus, the upper and lower 


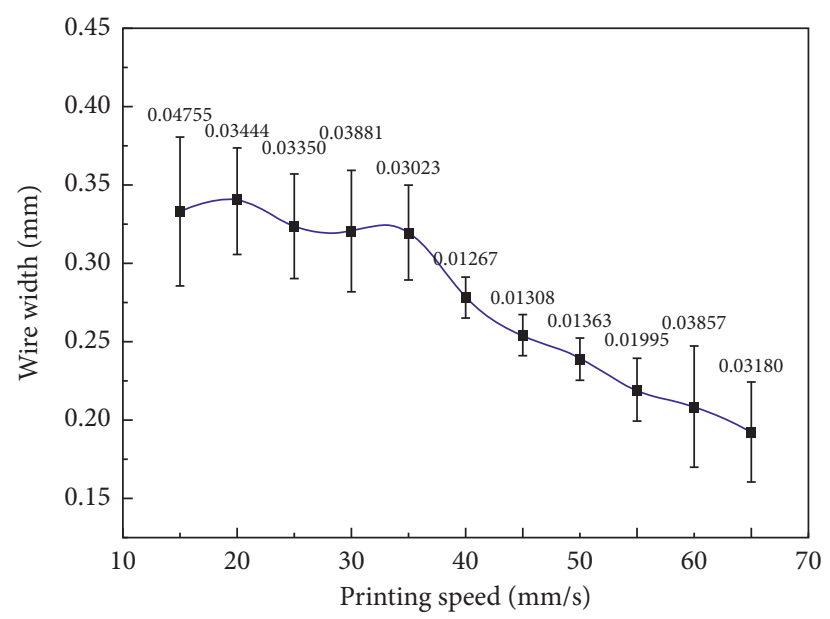

FIgURE 6: The effects of the printing speed on the Ga metal wire width.

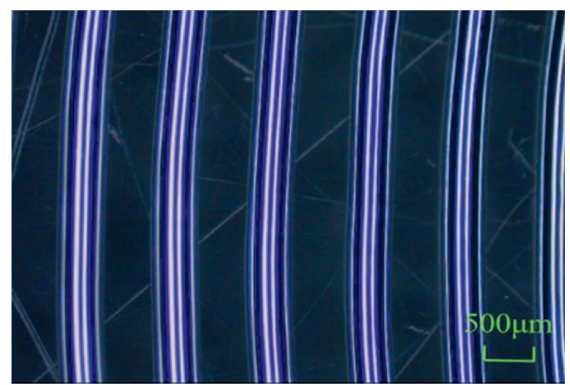

Figure 7: The optical micrograph result with the printing speed of $40 \mathrm{~mm} \cdot \mathrm{s}^{-1}$.

limit values of $W$ corresponding to different nozzle inner diameters $(d)$ are determined. The nozzle inner diameters of $d=0.11 \mathrm{~mm}, 0.21 \mathrm{~mm}, 0.31 \mathrm{~mm}, 0.41 \mathrm{~mm}, 0.51 \mathrm{~mm}$, and $0.61 \mathrm{~mm}$ are selected in the experiments. The effect of nozzle inner diameter on the $\mathrm{Ga}$ metal wire width is shown in Figure 8 . When $d=0.11 \mathrm{~mm}$, the corresponding minimum Ga wire width $W_{0.11 \text { min }}=0.032 \mathrm{~mm}$, as shown in Figure 9; when $d=0.61 \mathrm{~mm}$, the corresponding maximum width of Ga wire $W_{0.61 \text { max }}=0.343 \mathrm{~mm}$. The linear fitting of $W_{\text {min }}$, $W_{\max }$ shows that there are positive correlations between $W_{\min }$ and $d$ and $W_{\max }$ and $d$. Once the nozzle inner diameter $d$ is determined, the range of liquid Ga metal wire width can be preliminarily determined. On the contrary, the nozzle diameter can be determined according to the target value of wire width, and other parameters can be selected on this basis.

3.6. Effects of Sealing Process on the Printed Ga Metal Wire. The influence of sealing technology on liquid Ga metal is an important factor to determine the quality of finished products. The effects of the nozzle inner diameter on the Ga metal wire width before and after sealing process are shown in Figure 10. It should be noted that each Ga metal wire width is taken from the middle value of the forming range under the corresponding nozzle inner diameters. In order to compare the Ga metal wire width before and after sealing process, the linear fit of the values of Ga metal wire width before and after sealing process is implemented, respectively. According to Figure 10, it is considered that the aforementioned transferring and sealing process based on PVA film has almost no effect on the width of the Ga wire. It shows that the existence of oxide film on the surface of liquid $\mathrm{Ga}$ metal provides sufficient stability for the printed structure. To study the effects of sealing process on the performance of the printed Ga metal wire, the resistance per unit length with the different $\mathrm{Ga}$ metal wire widths before and after sealing is measured, as shown in Figure 11. In the same way, it should be noted that each Ga metal wire width is taken from the middle value of the forming range under the corresponding nozzle inner diameters. The linear fit of the values of the resistance per unit length before and after sealing process is implemented, respectively. It can be seen that the resistance per unit length before and after sealing process decreases when the Ga metal wire width increases. On the other hand, with the increase of Ga metal wire width, the influence of sealing process on the resistance per unit length decreases. From Figure 11, it can be seen that the resistance per unit length after sealing is larger than that before sealing. The reason is that the local stress concentration under the silica gel coating causes the cross-sectional area to decrease and the resistance per unit length to increase. When the nozzle inner diameter $d=0.61 \mathrm{~mm}$ (the middle value of the corresponding Ga metal width is about $0.265 \mathrm{~mm}$ ), the aforementioned transferring and sealing technology based on PVA film has almost no effect on the 


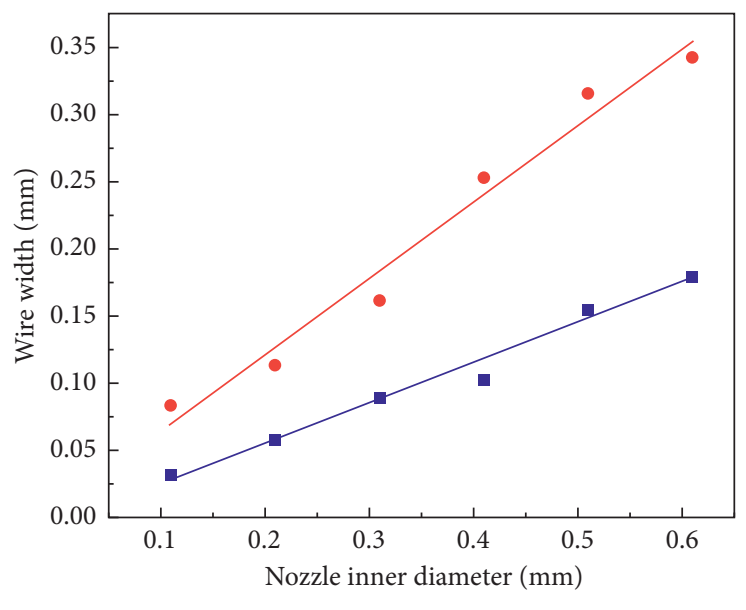

- Wmin

- Wmax

FIgURE 8: The effects of nozzle inner diameter on the Ga metal wire width.

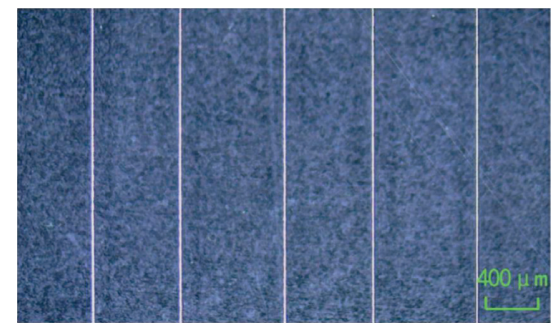

Figure 9: Optical micrograph of wire width of $0.032 \mathrm{~mm}$.

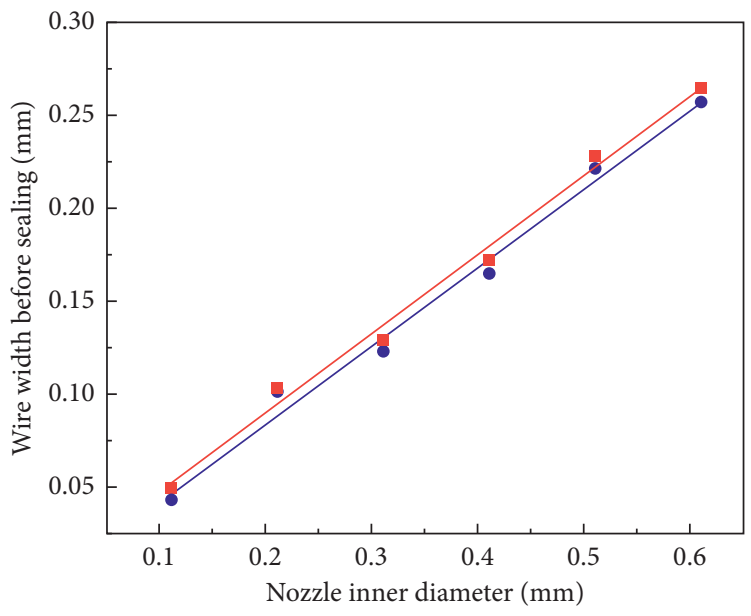

- Wire width before sealing

- Wire width after sealing

FIgURE 10: The effects of the nozzle inner diameter on the Ga metal wire width before and after sealing process.

resistance per unit length of the Ga metal wire. However, the silica gel solution does have some effect on the wire width and resistance during the pouring and sealing process. The optimized process parameters greatly improve the resistance ability of $\mathrm{Ga}$ wire to external pressure, and thus higher forming quality is obtained. According to the experimental results, when the Ga metal wire is printed using the nozzle whose inner diameter is less than $0.61 \mathrm{~mm}$, an appropriate increase of the Ga metal wire width can effectively improve the stability of resistance per unit length for the product. 


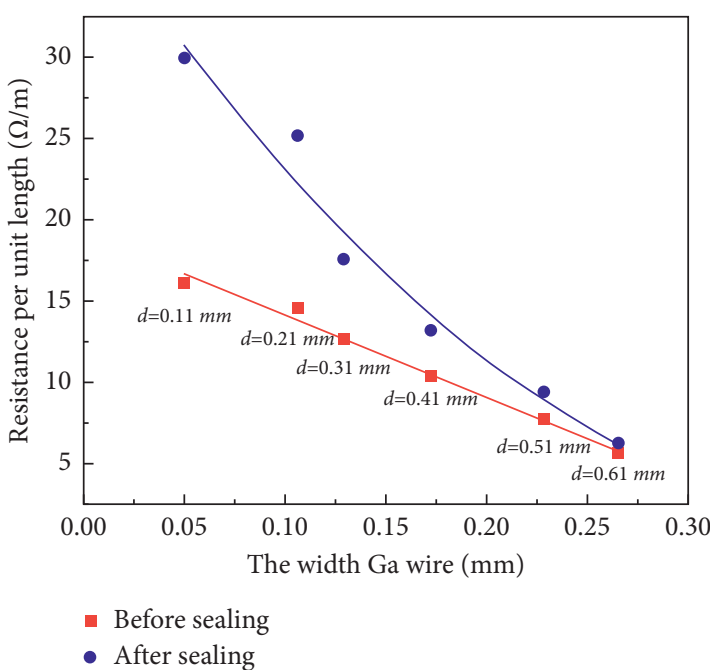

FIgURE 11: The effects of the Ga metal wire width on the resistance per unit length before and after sealing process.

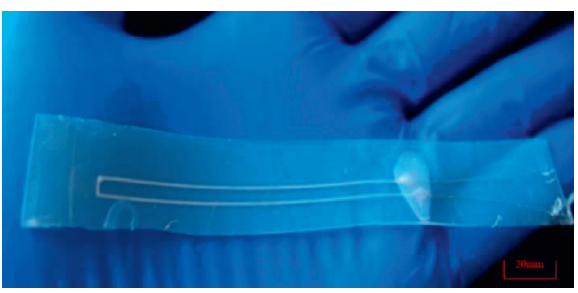

FIgURE 12: The flexible liquid Ga metal sensor.

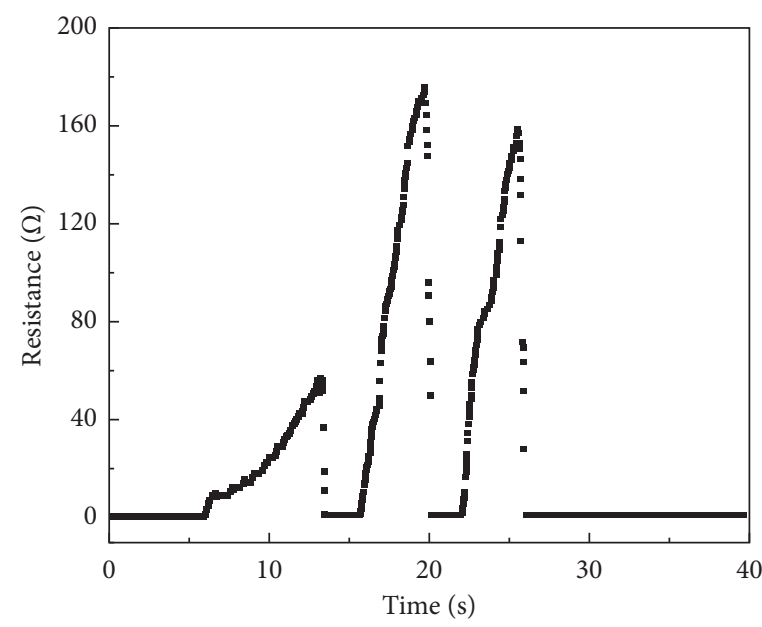

(a)

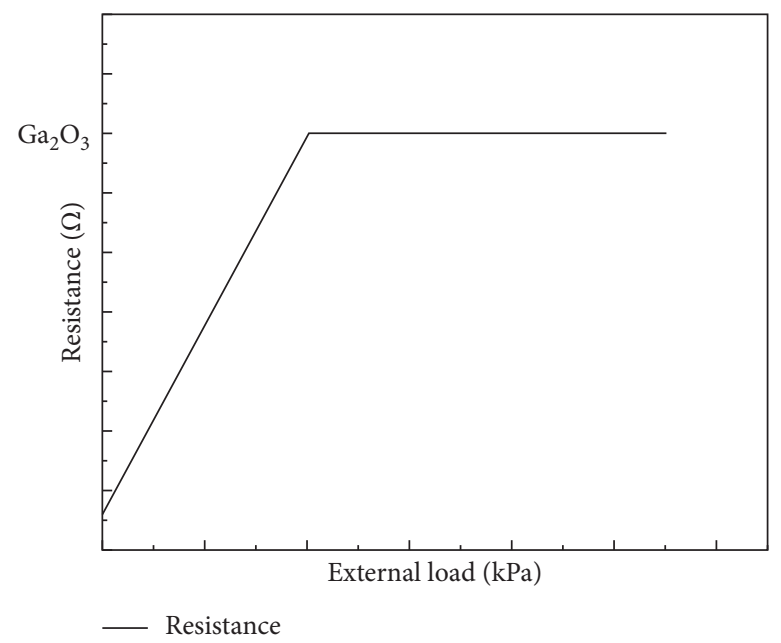

(b)

FIGURE 13: Electrical resistance response of a prepared flexible sensor to the external load. (a) Change of resistance with time under an external load. (b) Schematic diagram of relationship between the external load and resistance.

3.7. Flexible Resistance Sensor. The fabricated flexible sensor constructed after transferring and sealing is shown in Figure 12. Some features are represented through measuring the resistance of the flexible sensor. Considering the applications under the extreme environment, the resistance value is measured when the flexible sensor is stretched and then released to represent the situation under sudden external tensile. The experimental results are shown in Figure 13(a). For instance, the resistance value $R_{0}=1.346 \Omega$ when the initial state $t=0$. When the sensor is subjected to external load of $P=500 \mathrm{kPa}$ at $t=19.66 \mathrm{~s}$, the resistance value $R_{t=19.66}=176 \Omega$ and the resistance change rate $\Delta R /$ $R_{0}>12900 \%$. After removing the external load, the product resistance value recovers rapidly within $0.4 \mathrm{~s}$, and the phase 
difference value between the restored resistance value and $R_{0}$ is less than $0.01 \Omega$. It can be seen that the flexible sensor can immediately display the change of resistance value under the action of external load. The flexible sensor has a very wide measurement range and good restorative property under the external load. There is an oxidation film on the surface of liquid metal, which will be infiltrated by silica gel during the sealing process. Even under large deformation, the resistance of $\mathrm{Ga}_{2} \mathrm{O}_{3}$ will be displayed when the structure of $\mathrm{Ga}$ metal is disconnected. The maximum resistance value $R_{\max }$ is equal to the resistance value $R_{\mathrm{Ga}_{2} \mathrm{O}_{3}}$, as shown in Figure 13(b). Combined with the flow and low melting point characteristics of liquid Ga metal, the failure of the sensor caused by the fracture of Ga wire is avoided. Therefore, it can be used in some large deformation condition, such as the real-time pressure monitoring of human joints and the surface of the sole of the foot during strenuous exercise, or the front-end sensing of the detector in unknown environment.

\section{Conclusions}

In this paper, the direct writing process and flexible sealing technology of liquid Ga metal are studied. The results show that piston extrusion is suitable for the direct writing of liquid $\mathrm{Ga}$ metal compared to screw extrusion and pneumatic extrusion. The Ga metal width ranging from $0.032 \mathrm{~mm}$ to $0.343 \mathrm{~mm}$ by direct writing of liquid Ga metal is realized, which provides a certain guidance on the selection of relevant printing parameters. A transferring and sealing technology based on PVA film is proposed. The effects of sealing process on sensor performance are analysed. The experimental results showed that the Ga metal wire width is almost not affected by the silica gel sealing process, and when the Ga metal wire is printed using the nozzle whose inner diameter is $0.61 \mathrm{~mm}$, the Ga metal wire width is almost not affected by the silica gel sealing process. The flexible resistance sensor fabricated by this transferring and sealing technology has a wide measurement range, and the resistance change rate $\Delta R / R_{0}>12900 \%$. When the external load is removed, the resistance value of the flexible sensor can return to the initial state in $0.4 \mathrm{~s}$ and the phase difference value between the restored resistance value and $R_{0}$ is less than $0.01 \Omega$.

\section{Data Availability}

The data used to support the findings of this study are available from the corresponding author upon request.

\section{Conflicts of Interest}

The authors declare that they have no conflicts of interest regarding the publication of this paper.

\section{Acknowledgments}

This study was supported by the Science and Technology Program of Guangdong Province, China (2016B090915001), and 2018 Zhuhai City Industrial Core and Key Technology Research Direction Project (ZH01084702180085HJL).

\section{References}

[1] S. Wang, J. Y. Oh, J. Xu, H. Tran, and Z. Bao, "Skin-inspired electronics: an emerging paradigm," Accounts of Chemical Research, vol. 51, no. 5, pp. 1033-1045, 2018.

[2] J. Chen, J. Zhang, Z. Luo, and J. Zhang, "Super elastic, sensitive, and low hysteresis flexible strain sensor based on wavepatterned liquid metal for human activity monitoring," ACS Applied Materials \& Interfaces, vol. 12, no. 19, pp. 2220022211, 2020.

[3] P. A. Lopes, B. C. Santos, A. T. de Almeida, and M. Tavakoli, "Reversible polymer-gel transition for ultra-stretchable chipintegrated circuits through self-soldering and self-coating and self-healing," Nature Communications, vol. 12, no. 1, p. 4666, 2021.

[4] X. Qu, S. Wang, Y. Zhao et al., "Skin-inspired highly stretchable, tough and adhesive hydrogels for tissue-attached sensor," Chemical Engineering Journal, vol. 425, Article ID 131523, 2021.

[5] D. G. Marques, P. A. Lopes, A. T. De Almeida, C. Majidi, and M. Tavakoli, "Reliable interfaces for E-GaIn multi-layer, stretchable circuits and microelectronics," Lab on a Chip, vol. 19, no. 5, pp. 897-906, 2019.

[6] Z. Yu, G. Cai, P. Tong, and D. Tang, "Saw-toothed microstructure-based flexible pressure sensor as the signal readout for point-of-care immunoassay," ACS Sensors, vol. 4, no. 9, pp. 2272-2276, 2019.

[7] P. A. Lopes, D. Vaz Gomes, D. Green Marques, P. Faia, R. G. Joana, and J. Fernando Jord, "Soft bioelectronic stickers: selection and evaluation of skin-interfacing electrodes," Advanced Healthcare Materials, vol. 8, no. 15, Article ID 1900234, 2019.

[8] J. Alberto, C. Leal, C. Fernandes, and P. Lopes, "Fully untethered battery-free biomonitoring electronic tattoo with wireless energy harvesting," Scientific Reports, vol. 10, no. 1, p. 5539, 2020.

[9] Z. Yu, G. Cai, X. Liu, and D. Tang, "Platinum nanozymetriggered pressure-based immunoassay using a three-dimensional polypyrrole foam-based flexible pressure sensor," ACS Applied Materials \& Interfaces, vol. 12, no. 36, pp. 40133-40140, 2020.

[10] Z. Yu, Y. Tang, G. Cai, R. Ren, and D. Tang, "Paper electrodebased flexible pressure sensor for point-of-care immunoassay with digital multimeter," Analytical Chemistry, vol. 91, no. 2, 2018.

[11] H. Li, Y. Ma, Z. Liang, and Z. Wang, "Wearable skin-like optoelectronic systems with suppression of motion artifacts for cuff-less continuous blood pressure monitor," National Science Review, vol. 7, pp. 849-862, 2020.

[12] R. P. Rocha, P. A. Lopes, A. T. de Almeida, M. Tavakoli, and C. Majidi, "Fabrication and characterization of bending and pressure sensors for a soft prosthetic hand," Journal of Micromechanics and Microengineering, vol. 28, no. 3, Article ID 34001, 2018.

[13] M. Tavakoli, P. Lopes, J. Lourenço et al., "Autonomous selection of closing posture of a robotic hand through embodied soft matter capacitive sensors," IEEE Sensors Journal, vol. 17, no. 17 , pp. 5669-5677, 2017.

[14] A. Atalay, V. Sanchez, O. Atalay, and D. Vogt, "Batch fabrication of customizable silicone-textile composite capacitive strain sensors for human motion tracking," Advanced Materials Technologies, vol. 2, no. 9, 2017.

[15] S. Ali, D. Maddipatla, B. Narakathu, and A. Abdulmahdi Chlaihawi, "Flexible capacitive pressure sensor based on pdms 
substrate and ga-in liquid metal," IEEE Sensors Journal, vol. 19, no. 1, pp. 97-104, 2018.

[16] M. D. Dickey, "Emerging applications of liquid metals featuring surface oxides," ACS Applied Materials \& Interfaces, vol. 6, no. 21, pp. 18369-18379, 2014.

[17] Y. Park, B. Chen, and R. Wood, "Design and fabrication of soft artificial skin using embedded microchannels and liquid conductors," IEEE Sensors Journal, vol. 12, no. 8, pp. 2711-2718, 2012.

[18] J. Jiang, S. Zhang, B. Wang, H. Ding, and Z. Wu, "Hydroprinted liquid-alloy-based morphing electronics for fastgrowing/tender plants: from physiology monitoring to habit manipulation," Small, vol. 16, no. 39, Article ID 2003833, 2020.

[19] L. Zhou, Q. Gao, J. Zhan, C. Xie, J. Fu, and Y. He, "Threedimensional printed wearable sensors with liquid metals for detecting the pose of snakelike soft robots," ACS Applied Materials \& Interfaces, vol. 10, no. 27, pp. 23208-23217, 2018.

[20] A. D. Aguilar-Banegas, F. D. Reyes-Cruz, J. A. Vargas-Pineda, and C. H. Ortega-Jimenez, "Literature review of gallium: conductive ink alternative?" Materials Science Forum, vol. 975, pp. 139-144, 2020.

[21] M. J. Assael, I. J. Armyra, J. Brillo, S. Stankus, J. Wu, and W. Wakeham, "Reference data for the density and viscosity of liquid cadmium, cobalt, gallium, indium, mercury, silicon, thallium, and zinc," Journal of Physical and Chemical Reference Data, vol. 41, p. 3, 2012.

[22] R. C. Gough, A. M. Morishita, J. Dang, and M. Robert Moorefield, "Rapid electrocapillary deformation of liquid metal with reversible shape retention," Micro \& Nano Systems Letters, vol. 3, no. 1, p. 4, 2015.

[23] A. Fassler and C. Majidi, "3D structures of liquid-phase gain alloy embedded in PDMs with freeze casting," Lab on a Chip, vol. 13, no. 22, pp. 4442-4450, 2013.

[24] C. Jin, J. Zhang, X. Li, X. Yang, J. Li, and J. Liu, "Injectable 3-d fabrication of medical electronics at the target biological tissues," Scientific Reports, vol. 3, no. 1, pp. 1-7, 2013.

[25] Y. Zheng, J. Liu, Z. He, and Y. Gao, "Direct desktop printedcircuits-on-paper flexible electronics," Scientific Reports, vol. 3, no. 1, 2013.

[26] Y. Yamada, O. Sancakoglu, R. Sugiura, M. Shoriki, and S. Funaki, "Electrical resistivity reduction and spatial homogenization of Ga-doped ZnO film by Zn layer insertion," Thin Solid Films, vol. 707, Article ID 138069, 2020.

[27] G. Li and D. W. Lee, "An advanced selective liquid-metal plating technique for stretchable biosensor applications," Lab on a Chip, vol. 17, no. 20, pp. 3415-3421, 2017.

[28] Y. R. Jeong, J. Kim, Z. Xie et al., "A skin-attachable, stretchable integrated system based on liquid GaInSn for wireless human motion monitoring with multi-site sensing capabilities," NPG Asia Materials, vol. 9, no. 10, 2017.

[29] D. Kim, P. Thissen, V. Viner et al., "Recovery of nonwetting characteristics by surface modification of gallium-based liquid metal droplets using hydrochloric acid vapor," ACS Applied Materials \& Interfaces, vol. 5, no. 1, pp. 179-185, 2012.

[30] T. Kawanago, T. Matsuzaki, and S. Oda, "Transfer printing of gate dielectric and carrier doping with poly(vinyl-alcohol) coating to fabricate top-gate molybdenum disulfide field-effect transistors," Japanese Journal of Applied Physics, vol. 59, no. $12,2020$.
[31] M. Nisser, J. Zhu, T. Chen, K. Bulovic, P. Punpongsanon, and S. Mueller, "Sequential support: 3d printing dissolvable support material for Time-Dependent mechanisms," in Proceedings of the Thirteenth International Conference on Tangible, Embedded, and Embodied Interaction, pp. 669-676, New York, NY, USA, March 2019. 